1

\section{A PROPOSED COEFFICIENT OF SYMMETRY}

\author{
HANY A.MAKROUM"
}

Most frequency distributions obtained - in practice - from a limited set of readings are asymmetrical to a degree. Several measures of skewness (departure from symmetry) are available - in the literature. Probably the most accurate and suitable for :general use is the standardized third moment about the mean. In the literature,it is agreed that the third moment will be zero for a symmetric distribution, negative for skewness to the - left and positive for skewness to the right. However, the third. moment, can equal zero without the distribution being symmet- : rical. In other words, symmetry can not be expressed in terms of one or two moments - it requires an infinity of them.

:The proposed coefficient of symmetry presents the solution for this problem. For a distribution, if the proposed coefficient (ks) equals one then the associated distribution is perfectly symmetric. The values of ks less than unity indicate less sym-: :metry.

\title{
INTRODUCTION
}

:While we cannot characterize completely the probability distribution of a variable we do know some quantitative things about it. A natural way in which partial information may be :expressed is in terms of the moments of the distribution. - The rth moment of a distibution is the expected value of the rth power of the variable. The first moment of a distribution: .leads to the mean value if it is equated. to zero, and the second moment taken about the meam leads to the variance and the standard deviation. We will now consider the significance of the third moment about the mean. This is equal to

$$
\int_{b}^{a}(x-\mu)^{3} f(x) d x
$$

* Lecturer,Dpt. of Production Engineering, Helwan University, Cairo Egypt. 


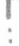

: for a continuous distribution $f(x)$ which exists between the limits a and $b$ where $\mu$ is the mean value. If only discrete readings are available, the third moment is given by

$\vdots$

$$
\sum_{i=1}^{i=n} \frac{\left(x_{i}-\bar{x}\right)}{n}=6\left(\overline{x_{i}-\bar{x}}\right)^{3}
$$

The so-called coefficient of skewness ( $\mathrm{sk}$ ) is defined as the : skewness which is given by (2) divided by the cube of the standard deviation $(\sigma)$ or its estimate, as appropriate. For a discrete experimental distribution derived from $n$ (large) : : readings it is equal to:

$\quad \quad s k=\frac{\left(\overline{x_{i}-\bar{x}}\right)^{3}}{n \sigma^{3}}$

$\vdots$

\section{SKEWNESS AND SYMMETRY}

: The more moments we know the more we know about the distribution. But, not all of the information we might have about the distribution can be simply summarized in terms of the moments :

! of the distribution. For example, we might know that the tolerance distribution was symmetrical about the mean - the probability of a tolerance of $(\bar{z}+k)$ equals the probability of a toil

: rance for $(\bar{z}-k)$, for all k.

Because of the absence of a measure for the symmetry one can classify the references into the following three categories:

I. They treated the skewnws without considering the symmetry (e.g. [2] )

II. They treated the skewness and discarded the symmetry

: as unmeasurable characteristic (e.g. [4]).

III. They considered the symmetry without enough accuracy $(e \cdot g \cdot,[1])$.

The coefficient of skewness, sk, will be zero for a symmetrical distribution. However, sk, can equal zero without the distribut-

: ion being symmetrical. Now, it may be the case that in practice if we think that the distribution is symmetrical we really mean that $\mathrm{sk}=0$.

\section{NUMERICAL ILLUSTRATION}

Consider a situation where 170 observations were taken from: a production line and coded as in Fig. 1.

: We have the mean $(\overline{\mathrm{X}})=0$

The third moment $=\frac{2088-2088}{170}=0$

i... 


\begin{tabular}{|l|l|}
\hline $\mathrm{CA}-18$ & 185 \\
\hline
\end{tabular}

6

\begin{tabular}{|rrrrrrrrrrrrrrrrr|}
\hline 0 & -8 & -1 & 1 & 1 & 0 & 1 & -7 & -1 & 1 & 0 & 1 & 0 & 1 & -6 & 1 & -1 \\
9 & 0 & 1 & 0 & 1 & -6 & -1 & 1 & 0 & 8 & 1 & 0 & 1 & -5 & -1 & 1 & 0 \\
1 & 0 & 1 & -5 & -1 & 1 & 0 & 7 & 0 & 1 & -5 & -1 & 1 & 0 & 1 & 0 & 1 \\
1 & -4 & -1 & 1 & 0 & 6 & 1 & 0 & 1 & -4 & -1 & 1 & 0 & 1 & 0 & 1 & -4 \\
-1 & 1 & 0 & 1 & 5 & 0 & 1 & -3 & -1 & 1 & 0 & 1 & 0 & 1 & -3 & -1 & 1 \\
0 & 4 & 1 & 0 & 3 & 1 & -3 & -1 & 1 & 0 & 1 & 0 & -3 & -1 & 1 & 0 & 1 \\
0 & -3 & -1 & 1 & 0 & 1 & 0 & -3 & -1 & 1 & 0 & 1 & 2 & 0 & -2 & -1 & 1 \\
0 & 1 & 0 & -2 & -1 & 1 & 0 & 1 & 0 & -2 & -1 & 1 & 0 & 1 & 0 & -2 & -1 \\
1 & 0 & 1 & 0 & -2 & -1 & 1 & 0 & 1 & 0 & -2 & -1 & 1 & 0 & 1 & 0 & 1 \\
1 & 0 & 1 & 0 & -1 & 1 & 0 & 1 & 0 & 1 & 0 & 1 & 0 & 1 & 0 & 1 & 0
\end{tabular}

Fig. 1 .

$\vdots$

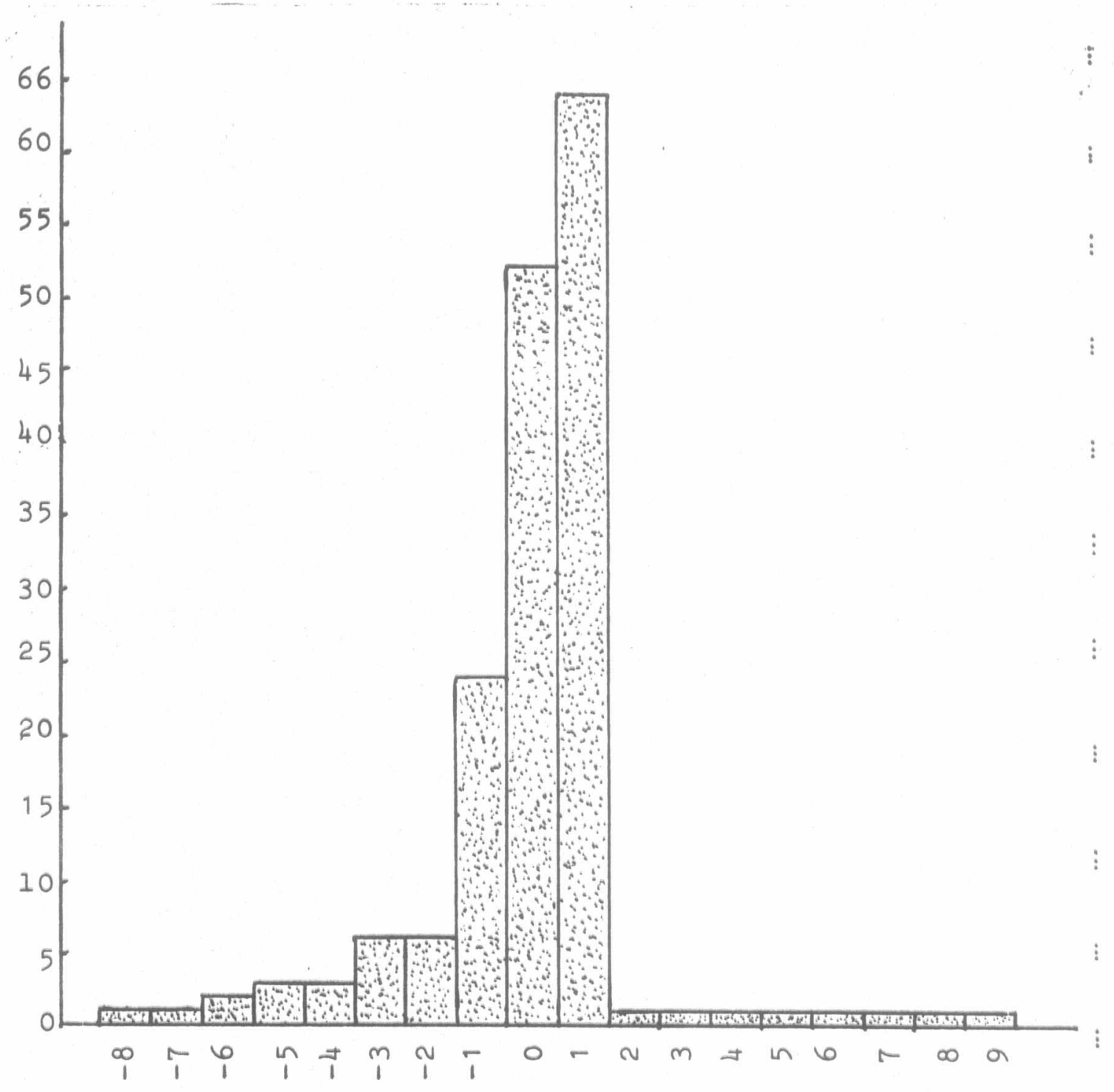

Fig. 2. Histogram of data given in Fig. 1. 
: The coefficient of skewness $=0 / \sigma^{3}=0$

The frequency distribution of the ata (Fig.2) is clearly : asymmetrical (skewed), although the first and third moments are zeros.

\section{SYMMETRY CONDITION}

Symmetry condition states that if the mean values of each two : conjugate readings thave the same order below and above the median), in an ordered data, are equal then the associated distribution is perfectly symmetric.

\section{DERIVATION OF THE PROPOSED COEFFICIENT OF SYMMETRY}

The root mean square deviation of any two conjugate readings $X_{j}$ and $X_{j}$ about any point deviated by $Y_{j}$ from their arithmetic mean $\overline{\mathrm{X}}_{j} \mathrm{~J}_{\text {is }} \mathrm{given}$ by

$\vdots \quad \sqrt{\frac{\left(x_{j}-X_{j^{\prime}}\right)^{2}}{4}+Y_{j}^{2}}$

The root mean square deviation (standard) of all readings

$$
\sigma=\sqrt{\sum_{j=1}^{n / 2} \frac{\left(x_{j}-x_{j}\right)^{2} / 4+\sum_{j=1}^{n / 2}\left(\bar{x}_{j}-\bar{x}\right)^{2}}{j}}=\sqrt{\frac{\sum_{i=1}^{n}\left(x_{i}-\bar{x}\right)^{2}}{n}}
$$

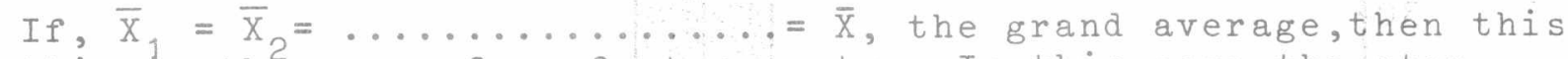
this is the case of perfect symmetry. In this case the stan: dard deviation (eq.4) reaches its minimum value.

$$
\therefore \quad \sigma_{\min }=\sqrt{\frac{\sum_{j=1}^{n / 2}\left(x_{j}-x_{j}\right)^{2}}{2 n}}
$$

The proposed coefficent of symmetry (ks) is given by (5) : divided by (4) as appropriate

$$
\therefore \quad k s=\sqrt{\sum_{j=1}^{n / 2}\left(x_{j}-x_{j}\right)^{2} / 2 \sum_{i=1}^{n}\left(x_{i}-\bar{x}\right)^{2}}
$$

¿.. 


\section{APPLCIATION TO THE NUMERICAL EXAMPLE}

Considering the same data of Fig。 1 , we order the data ascen! dingly as indicated below.

$$
\begin{aligned}
& \mathrm{x}_{1} \mathrm{x}_{2} \mathrm{x}_{3} \mathrm{x}_{4} \mathrm{x}_{5} \mathrm{x}_{6} \ldots \ldots \mathrm{x}_{6}^{\prime} \mathrm{x}_{7}^{\prime} \mathrm{x}_{4}^{\prime} \mathrm{x}_{3}^{\prime} \mathrm{x}_{2}^{\prime} \mathrm{x}_{1}^{\prime}
\end{aligned}
$$

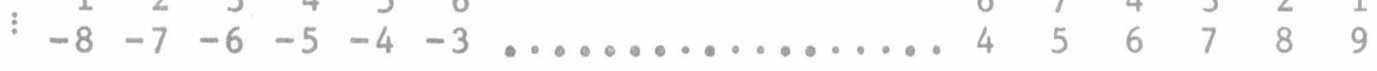

$$
\begin{aligned}
& \vdots \quad \mathrm{ks}=\frac{14.3}{2 \times 7.58}=0.971 \text {. }
\end{aligned}
$$

The value of $\mathrm{ks}$ for our data is 0.971 , which is clear indication of asymmetry.

\section{CONCLUSIONS}

: In this paper we have presented a proposed simple coefficient of symmetry (ks), and the first symmetry condition. The proposed coefficient is the first measure and solution for the : problem of symmetry which can not be expressed in temrs of large number of moments. Because of the abscence of such measure, the contributers (e.g。, [4]) had lost the symmetry as a useful characteristic. In the light of this paper the symmetry can take its position as an important characteristic expressible in terms of a simple and clear measure.

$\vdots$

\section{REFERNCES}

: 1. Besterfie1d, D.H.," Auality Control". Prentice-Hall, Inc., Englewood Cliffs, (1979).

: 2. Croxton,F.E. Cowden, D.J。 and K1ein S., "Applied General Statistics" Prentice-Hall of India, New Delhi, (1979). 3. Dietrich,C,F."Urcertainty, Calibration and Probability".
Adam Hilger, London, (1973).

4. Starr, $\mathrm{M}_{,} \mathrm{K}_{e}$, and Miller, D.W., "Inventory Control, Theory and: Practice", Prentice-Ha11 of India, New Delhi,(1974). 
